\title{
Uma Solução Cooperative Positioning Integrada com Dead Reckoning para Suporte a GPS Sem Linhas de Visada Utilizando a Tecnologia de Radio 802.11p
}

\author{
Pedro Paulo Libório Lima do Nascimento ${ }^{1}$, Bruno Yuji Lino Kimura ${ }^{2}$, \\ Daniel Ludovico Guidoni ${ }^{3}$, Leandro Aparecido Villas ${ }^{1}$ \\ ${ }^{1}$ Instituto de Computação - UNICAMP \\ ${ }^{2}$ Instituto de Ciência e Tecnologia - UNIFESP \\ ${ }^{3}$ Departamento de Ciência da Computação - UFSJ \\ liborio@lrc.ic.unicamp.br, bruno.kimura@unifesp.br \\ guidoniaufsj.edu.br, leandrodic.unicamp.br
}

\begin{abstract}
In Intelligent Transportation Systems (ITS) and Vehicular Networks (VANETS), a myriad of applications has been developed in the last years with different requirements of location accuracy. The vast majority of such applications use the Global Positioning System (GPS) to obtain the device's current location. However, in the absence of a satellite line-of-sight, a typical situation in places such as tunnels, multilevel highways and dense urban areas, the GPS signal is exposed to multipath effect, causing severe loss of location information in the receiver. In this paper, we characterize the problem of GPS unavailability in urban areas from datasets of movements of buses and taxis. To solve such a problem, we propose $\mathrm{CP}$ - an integrated solution for Cooperative Positioning based on three mechanisms: Dead Reckoning-based navigation; dissemination of location in VANET and node selection by Geometric Dilution of Precision; and map adjustment.
\end{abstract}

Resumo. Em Sistemas de Transporte Inteligentes (ITS) e Redes Veiculares (VANETs), uma miríade de aplicações foi desenvolvida nos últimos anos com diferentes exigências de acurácia de localização. A grande maioria dessas aplicações utiliza o Sistema de Posicionamento Global (GPS) para obter a localização atual do dispositivo. Entretanto, na ausência de linha de visada para satélites, situação típica em locais como túneis, rodovias multinível e áreas urbanas densas, o sinal GPS fica exposto ao efeito de multi-caminhos, causando severa perda de informação de localização no receptor. Neste trabalho, caracterizamos o problema da indisponibilidade do sistema GPS em áreas urbanas a partir de conjuntos de dados reais de deslocamentos de ônibus e táxis. Para tal problema, propomos $\mathrm{CP}$ - uma solução integrada de posicionamento cooperativo baseada em três mecanismos: navegação estimada por sensores Dead Reckoning; disseminação de localização em rede veicular e seleção de nós por geometria de diluição de precisão; e ajuste de mapa. Resultados de simulações mostram que a solução proposta possibilita desempenho superior ao GPS e ao GPS integrado ao Dead Reckoning.

\section{Introdução}

Os sistemas de tráfego evoluíram com o crescimento dos centros urbanos, possibilitando melhorias no transporte público e privado de pessoas, bens e serviços. No entanto, 
maiores problemas surgiram proporcionalmente a esse crescimento, como os congestionamentos, gerenciamento do tráfego e controle e prevenção de acidentes. Segundo o relatório da Organização Mundial de Saúde (OMS), em 2015 cerca de 1,25 milhão de pessoas morreram devido a acidentes de trânsito, gerando um custo governamental de aproximadamente 3\% do PIB dos países [OMS 2015]. Para solucionar esses problemas, os Sistemas de Transportes Inteligentes (do inglês, Intelligent Transportation Systems ITS) visam prover metodologias de comunicação, processamento e armazenamento de dados voltados para o segmento de transportes e comutação de pessoas nas cidades.

Para o pleno funcionamento de aplicações, serviços e protocolos para ITS é necessário algum mecanismo de localização dos elementos de transporte. A solução imediata de localização é o uso de um receptor GPS. No entanto, o sistema GPS apresenta problemas indesejáveis de operação em áreas urbanas densas, zonas de vasta vegetação, rodovias multinível e túneis. Nesses cenários críticos, onde há oclusão total ou parcial de linha de visada entre os satélites e receptores GPS, os sinais sofrem consequência do efeito de multi-caminhos. Em tal situação, os sinais de GPS são refletidos ou refratados por obstáculos, como prédios e materiais diversos, chegando deformado no receptor e, consequentemente, causando um aumento significativo do erro na estimativa de posicionamento. Em túneis, o sistema GPS possui seu pior desempenho devido a total oclusão da linha de visada, o que torna o serviço indisponível. Essas características do sistema GPS impõem desafios para o protocolos, aplicações e serviços em ITS, os quais requerem contínua e precisa informação de localização dos seus elementos de transporte.

Neste trabalho, propomos CP (Cooperative Positioning) - uma solução integrada de posicionamento cooperativo para resolver o problema de localização em cenários críticos para o sistema GPS. Para cobrir as zonas de indisponibilidade do GPS, a solução estima o posicionamento do dispositivo através de sensores DR (Dead Reckoning) e aproveita o potencial das VANETs para disseminar as estimativas entre os elementos de transporte. A solução CP calibra os sensores DR periodicamente e faz uso da geometria dos nós para selecionar os nós com melhor capacidade de localização. Por meio de um mapa digital MA (Map Adjustment), CP ajusta a estimativa final de posicionamento às vias de tráfego dos veículos, utilizando as informações de geometria da pista. A comunicação prevista ocorre por meio do padrão IEEE $802.11 \mathrm{p}$ em rede puramente Veículo-para-Veículo (V2V). Para validar a solução proposta, selecionamos conjuntos de dados reais de sinais GPS coletados por ônibus e táxis em regiões de túneis de grandes centros urbanos, onde ocorrem os problemas de indisponibilidade do GPS. A partir dos conjuntos de dados, reproduzimos as situações de indisponibilidade em ambiente de simulação e aplicamos as técnicas de localização desenvolvidas.

Este trabalho está organizado como segue. A Seção 2 apresenta os trabalhos relacionados. Os fundamentos dos sistemas de localização são apresentados na seção 3. A Seção 4 apresenta a solução proposta para o problema de localização chamada Cooperative Positioning. Os resultados e os trabalhos futuros são apresentados nas Seções 5 e 6 , respectivamente.

\section{Trabalhos Relacionados}

Soluções de localização propostas na literatura para VANETs e ITS podem ser divididas em duas categorias: soluções que utilizam a rede veicular para assistir os sistemas GPS; soluções que não utilizam sistemas GPS. Na primeira categoria, as soluções propostas [Alam and Dempster 2013], [Drawil and Basir 2010],[Parker and Valaee 2007] e 
[Rohani et al. 2015] combinam as informações fornecidas por receptores GPS com as informações cinemáticas dos veículos. Através de técnicas baseadas no alcance do sinal, como TOA (Time of Arrival), RSSI (Received Signal Strength Indicator) e RTT (Round Trip Time), esses trabalhos relatam acurácia entre 3 e 5 metros no posicionamento estimado dos veículos. Na segunda categoria, para substituir o sistema GPS, as soluções propostas empregam tecnologias de transmissão de radio-frequência com ou sem o suporte de infraestrutura de RSUs (Road Side Units) [Wahab et al. 2013] [Sun et al. 2012]. No entanto, como reportado por [Alam and Dempster 2013], as características inerentes às redes veiculares impõem desafios para abordagens não assistidas por GPS. Além disso, o uso de RSUs em larga escala em uma VANET pode ser custoso ${ }^{1}$.

Em [Parker and Valaee 2007], os autores propõem um algoritmo baseado em dois estágios: um de inicialização e outro de refinamento. Na inicialização, os veículos trocam as posições obtidas por GPS via trilateração. No refinamento, medidas de RSSI são utilizadas para estimar a distância entre os veículos. Ao final, um filtro de Kalman estendido é aplicado para associar os dados cinemáticos do veículo com os dados do sistema GPS.

Em [Alotaibi et al. 2014], os autores propõem RECOP (Relative Distributed AdHoc Localization). RECOP faz uso das distâncias entre os veículos, ângulos de chegada do sinal (Angle of Arrival (AOA)) da transmissão dos beacons e informações cinemáticas para construção de um mapa digital relativo. Através da troca dos mapas os veículos determinam sua posição. No entanto, o número de mapas cresce drasticamente conforme o aumento da densidade da rede. Informações de como a posição inicial dos veículos é determinada não são fornecidas.

Em [Alam and Dempster 2013], os autores consideram uma constante disponibilidade do sistema GPS. Os autores propõem uma abordagem onde os veículos são utilizados como estações base DGPS e trocam informações de localização utilizando a VANET. Resultados de simulações mostraram acurácia de 5 metros na localização. De forma similar, em [Rohani et al. 2015], os autores realizam fusão dos dados GPS e RSSI utilizando um Filtro de Kalman estendido, o que possibilitou melhorar acurácia de 6,6 metros do sistema GPS para 3 metros.

Em [Hoang et al. 2017] os autores propõem a fusão de informações de distância obtidas através de rádios Ultra Wide Band - UWB e utilizam fusão de dados para mesclar as informações de GPS com as informações de rádios UWB provenientes dos veículos e RSUs. No entanto, os autores recorrem ao uso de RSUs para aprimorar a capacidade de localização dentro dos túneis além de utilizar em uma pista retilínea o que facilita $o$ modelo de mobilidade. RMSEs finais na faixa de 2 metros foram obtidos.

\section{Fundamentos de Sistemas de Localização}

\subsection{Sensores de Posicionamento}

Um odômetro provê a informação da distância percorrida pelo veículo. Um compasso digital ou um mapa avançado fornece o ângulo absoluto do veículo a partir da integração das rotações de um giroscópio. Com essas informações, a posição de um veículo em movimento pode ser estimada [Boukerche et al. 2008] [Skog and Handel 2009].

Giroscópios Micro Eletromecânicos (MEMS) são amplamente utilizados em sistemas de navegação, smartphones e câmeras digitais. Um giroscópio pode ser represen-

\footnotetext{
${ }^{1} \mathrm{O}$ custo médio de uma RSU é de cerca de USD 9.000,00
} 
tado pela Equação 1 [Woodman 2007]:

$$
\hat{\delta}_{i}=\delta_{i}+\sigma \sqrt{\Delta_{t}}+\beta_{\text {instability }}
$$

Onde $\delta_{i}$ é a taxa real de variação angular. O ruído é uma variável aleatória com média zero, cujo desvio padrão $\sigma$ cresce proporcionalmente à raiz quadrada do tempo $\Delta_{t}$ em segundos, o efeito deste ruído é denominado random walk [Woodman 2007]. $\beta_{\text {instability }}$ é o desvio das estimativas ao longo do tempo. Como resultado, tem-se a estimativa $\hat{\delta}_{i}$.

Um odômetro mensura a distância a partir do número de rotações realizadas pela roda do veículo por meio de um enconder [Lawlor 1991]. A estimativa de erro do odômetro pode ser determinada por: $\epsilon_{\text {odometer }}=\frac{\left|\delta_{\text {actual }}-\delta_{\text {indicated }}\right| \times 100}{\delta_{\text {actual }}}$. Onde $\delta_{\text {actual }} \mathrm{e}$ $\delta_{\text {indicated }}$ são respectivamente a distância mensurada pelo odômetro e a distância real percorrida pelo veículo.

Um compasso digital é um dispositivo construído por magnetômetros, sendo responsável por fornecer medidas relativas ao norte magnético terrestre. Como alternativa ao compasso digital pode ser utilizado um mapa avançado [Skog and Handel 2009].

\subsection{Dead Reckoning (DR)}

DR é o processo de calcular a posição atual do veículo com base na última posição. A posição atual, contudo, é estimada a partir de medições dos sensores de posicionamento do veículo. O processo DR é ilustrado na Figura 1 e descrito no Algoritmo 1, que opera enquanto o sistema GPS estiver indisponível. O ponto $\left(x_{0}, y_{0}\right)$ representa a posição inicial do veículo fornecida via GPS no tempo $t_{0}$. O ponto $\left(x_{i}, y_{i}\right)$ é a posição estimada no tempo $i$. A variável $s_{i}$ representa a distância euclidiana percorrida pelo veículo a partir do ponto $\left(x_{i-1}, y_{i-1}\right)$ no tempo $t_{i-1}$. A variação angular relativa $\left(\hat{\delta}_{k}\right)$ é definida a partir da diferença entre duas leituras consecutivas DR. O ângulo absoluto $\theta_{i}$ no tempo $t_{i}$ assume as variações angulares $\hat{\delta}_{k}$ acumulada nos instantes $t_{1}, t_{2}, \ldots, t_{i}$. No ambiente de simulação as variáveis $\delta_{k}$ e $s_{i}$ simulam, respectivamente, o efeito de um giroscópio e um odômetro. A variação angular absoluta $\theta_{i}$ simula o efeito de um magnetômetro.

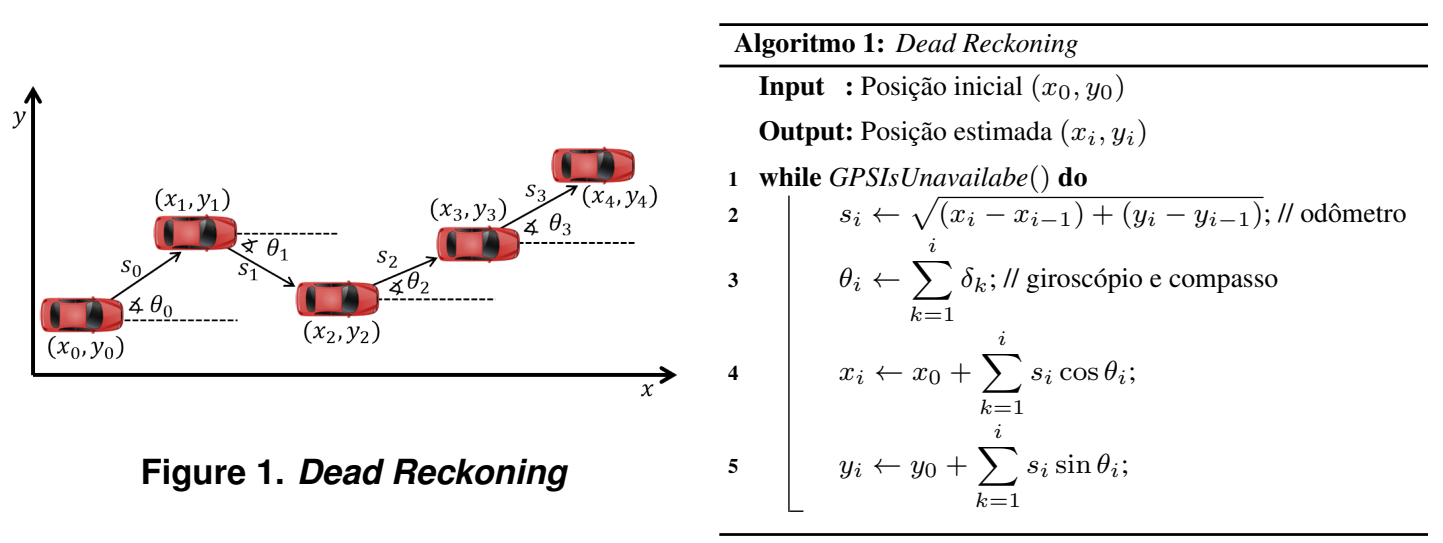

\subsection{Multilateração}

Na técnica de radio multilateração, o nó que deseja descobrir sua localização utiliza as posições fornecidas pelos nós vizinhos, e a informação da estimativa de distância obtida 
utilizando o Received Signal Strength Indicator (RSSI) [Parker and Valaee 2007] ou outras técnicas como TOA, TDOA ou AOA. Neste trabalho, utilizamos a técnica RSSI, uma vez que não é necessário nenhum hardware adicional.

Seja o conjunto de coordenadas dos $n$ nós vizinhos, $\left(x_{i}, y_{i}\right), i=0,1, . ., n$ e suas respectivas distâncias $r_{i}$ em relação ao nó que necessita estimar sua posição de coordenadas desconhecidas $\left(x_{u}, y_{u}\right)$. Portanto, têm-se o sistema de Equações cuja forma matricial é descrita na Equação 2.

$$
2\left[\begin{array}{cc}
\left(x_{n}-x_{0}\right) & \left(y_{n}-y_{0}\right) \\
\left(x_{n}-x_{1}\right) & \left(y_{n}-y_{1}\right) \\
\vdots & \vdots \\
\left(x_{n}-x_{n-1}\right) & \left(y_{n}-y_{n-1}\right)
\end{array}\right]\left[\begin{array}{l}
x_{u} \\
y_{u}
\end{array}\right]=\left[\begin{array}{ccccc}
\left(r_{0}^{2}-r_{n}^{2}\right) & + & \left(x_{0}^{2}-x_{n}^{2}\right) & + & \left(y_{0}^{2}-y_{n}^{2}\right) \\
\left(r_{1}^{2}-r_{n}^{2}\right) & + & \left(x_{1}^{2}-x_{n}^{2}\right) & + & \left(y_{1}^{2}-y_{n}^{2}\right) \\
\vdots & & \vdots & & \vdots \\
\left(r_{n-1}^{2}-r_{n}^{2}\right) & + & \left(x_{n-1}^{2}-x_{n}^{2}\right) & + & \left(y_{n-1}^{2}-y_{n}^{2}\right)
\end{array}\right]
$$

Este sistema de equações possui a forma $A x=b$ e possui várias soluções (possível indeterminado). Para solucioná-lo, basta aplicar o método (Least Squares) via decomposição QR [Sauer 2011]. Como resultado, é dado as coordenadas do nó desconhecido $\left(x_{u}, y_{u}\right)$ quando $i \geqslant 3$. De forma geométrica, a solução Least Squares encontra a intersecção entre os círculos estimando a posição do nós que deseja aprimorar ou descobrir sua posição.

\section{Solução Cooperative Positioning}

Ao contrário das técnicas não cooperativas (onde cada nó estima sua própria localização), a técnica de localização Cooperative Positioning - CP tem como objetivo o compartilhamento de métricas de localização entre veículos, tais como: erro observado, estimativa de localização e dados cinemáticos dos veículos. Neste sentido, um algoritmo distribuído pode ser implementado para melhorar a acurácia de localização. A técnica CP proposta é dividida em três estágios:

- Fase de Mensuração e Troca de Informações: os veículos coletam as informações cinemáticas e estimava de posição dos seus vizinhos. Essas informações são enviadas para os vizinhos via beacons periódicos.

- Fase de Localização: cada veículo aplica princípios de multilateração. Como resultado, têm-se uma matriz com as posições dos vizinhos e as distâncias informadas. Nessa etapa, o veículo estima a sua posição.

- Map Adjustment: cada veículo, utilizando a estimativa de sua posição, ajusta a sua posição na via de acordo com o mapa digital das vias.

A solução Cooperative Positioning proposta possui as seguintes características: i) Realizar reinícios periódicos na solução Dead Reckoning (cujo o erro cresce proporcionalmente ao tempo de uso [Skog and Handel 2009][Boukerche et al. 2008]) por meio das posições recebidas via multilateração ii) As informações de geometria da pista fornecida por um mapa digital avançado servirão para reajustar a posição Dead Reckoning às vias de tráfego dos veículos (Map Adjustment). iii) Como a solução proposta não utiliza nenhuma infraestrutura de comunicação auxiliar, foi utilizada a comunicação V2V entre veículos em uma abordagem multi-saltos, onde veículos que estão na entrada/saída do túnel, e possuem uma posição pelo GPS, possam enviar essa informação para os veículos dentro 
do túnel. Neste caso, apenas os veículos fora do túnel enviam beacons periódicos. Essa estratégia foi utilizada para que erros nas estimativas de posição não fossem utilizados pelos veículos.

\subsection{Envio e Descarte de Beacons}

Se um novo beacon de um mesmo veículo é recebido, as informações de posicionamento deste veículo são atualizadas em uma lista de nós vizinhos. Se um veículo vizinho não enviar um novo beacon abaixo de um tempo limite de espera, o nó é removido da lista de vizinhos. Isso é feito para que informações antigas não sejam utilizadas.

O Algoritmo 2 apresenta a solução para descarte de beacons antigos. Como entrada, o algoritmo recebe um tempo máximo de permanência das informações de um beacon na lista de nós vizinhos. A linha 1 faz a iteração por todos os nós vizinhos presentes na lista. A linha 2 calcula um valor delta que corresponde a diferença entre o tempo atual e o tempo em que o beacon foi enviado (timestamp). Na linha 3, é verificado se o beacon ultrapassou o tempo máximo para descarte. Em caso afirmativo, a linha 4 remove o beacon associado ao seu respectivo nó vizinho. Após a iteração por todos os nós vizinhos têm-se a lista de vizinhos atualizada que será utilizada para a estimativa de posição.

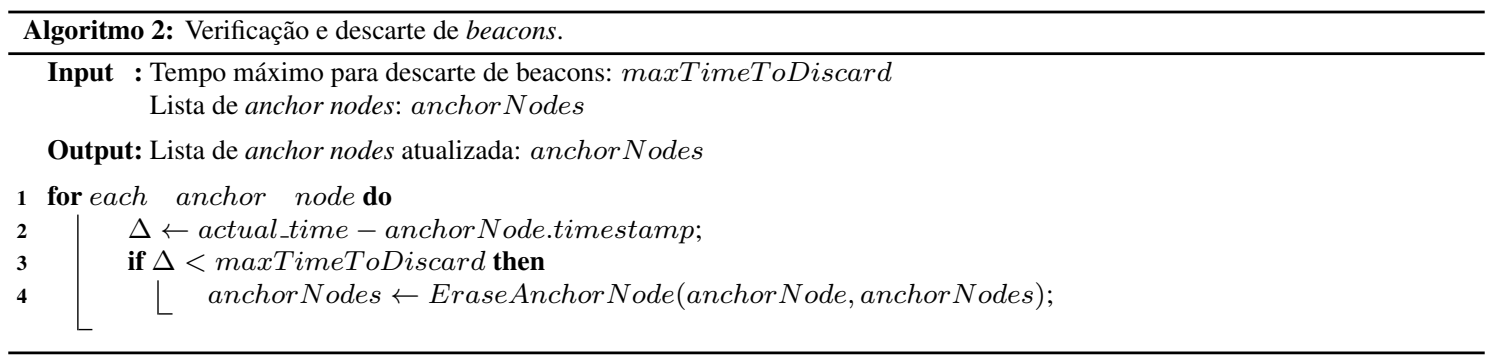

\subsection{Estimativa de Posição}

A estimativa de posição neste trabalho considera o problema da geometria dos nós utilizados no processo de multilateração (Subseção 3.3). O algoritmo proposto utiliza como base um conceito aplicado na seleção de satélites GPS, conhecido como Geometria de Diluição da Precisão (do inglês, Geometric Dilution of Precision - GDOP). Basicamente, o GDOP é uma métrica que avalia a influência da geometria dos satélites na estimativa de posicionamento do receptor GPS. Maiores detalhes sobre este problema podem ser encontrados em [Swaszek et al. 2017].

Considerando as $n$ posições dos nós vizinhos, $\left(x_{i}, y_{i}\right)$, suas respectivas distancias $r_{i}$ e para todo veículo $i=1, . ., n$ presente na lista de vizinhos do nó que deseja estimar/melhorar a sua posição, aplicamos a técnica de multilateração. Seja a posição estimada $\left(x_{u}, y_{u}\right)$ e a distância a partir de $\left(x_{u}, y_{u}\right)$ ao nó $\left(x_{i}, y_{i}\right)$ de $R_{i}=$ $\sqrt{\left(x_{u}-x_{i}\right)^{2}+\left(y_{u}-y_{i}\right)^{2}}$. A acurácia da estimativa via multilateração pode ser descrita estatisticamente pela matriz de covariâncias que é proporcional a inversa de $G^{T} G$. Onde $G$ possui a forma da Equação 3 e $G^{T}$ é a transposta de $G$. Um escalar é utilizado para avaliar o GDOP e seu valor equivale a Equação 4.

$$
G=\left[\begin{array}{cc}
\frac{x_{u}-x_{1}}{R_{1}} & \frac{y_{u}-y_{1}}{R_{1}} \\
\frac{x_{u}-x_{2}}{R_{2}} & \frac{y_{u}-y_{2}}{R_{2}} \\
\vdots & \vdots \\
\frac{x_{u}-x_{n}}{R_{n}} & \frac{y_{u}-y_{n}}{R_{n}}
\end{array}\right]
$$

$$
G D O P=\sqrt{\operatorname{trace}\left(G^{T} G\right)^{-1}}
$$




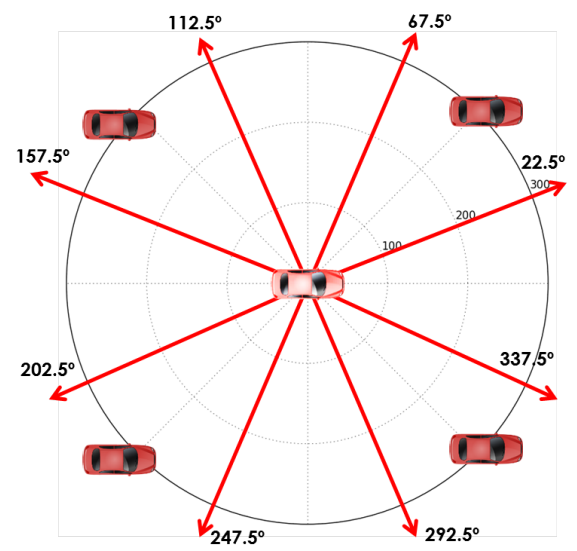

Figure 2. Seleção de nós baseadas em Ângulos. Neste Exemplo $n=4$ e $k=3$.

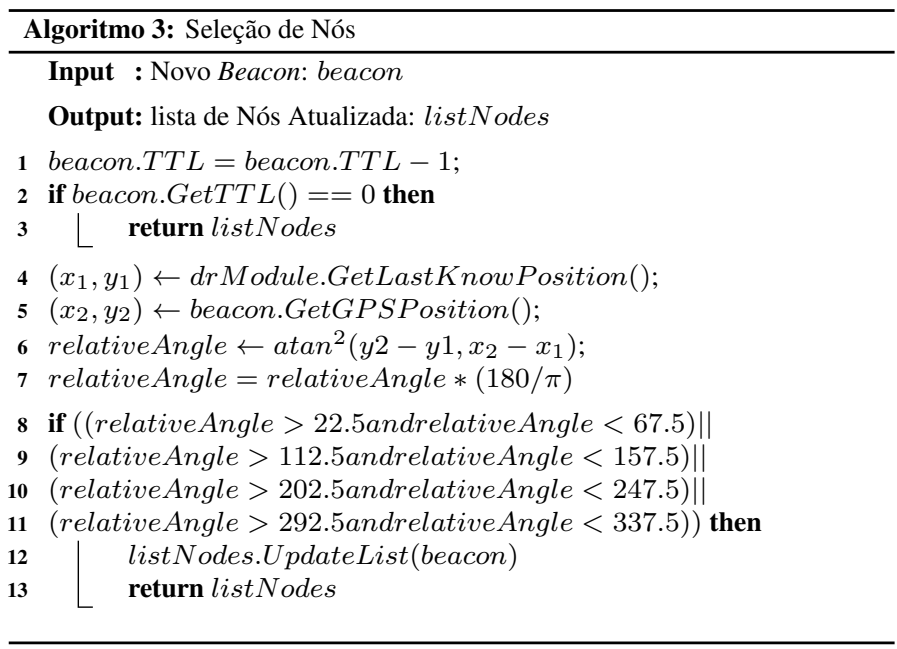

O GDOP equivale a raiz da soma dos elementos da diagonal principal de $\left(G^{T} G\right)^{-1}$. Quanto menor o valor do GDOP, melhor é a geometria dos nós selecionados para a multilateração [Swaszek et al. 2017]. Se há uma quantidade $n>3$ nós, existem diferentes combinações de nós (com diferentes geometrias) e uma destas combinações possui menor GDOP. Neste caso, escolher os nós com melhor geometria se torna um problema de combinatória e a quantidade de combinações equivale a $C\left(\begin{array}{l}n \\ k\end{array}\right)=\frac{n !}{k !(n-k) !}$. Outra questão importante é a alta colinearidade dos nós, causada principalmente pela topologia das pistas onde os veículos trafegam, o que causa um mal condicionamento da matriz de multilateração [Sauer 2011].

Devido a estes problemas e, visando diminuir a quantidade de combinações para avaliação do GDOP, também propomos a seleção de nós baseada nos ângulos relativos observados entre o nó que deseja aprimorar sua posição e seus vizinhos. Além disso, as combinações são tomadas sempre com $k=3$ (quantidade mínima de nós para uma multilateração). Esta seleção segue a mesma intuição observada na seleção de satélites GPS, onde a melhor distribuição geométrica dos satélites forma um tetraedro com maior volume [Swaszek et al. 2017]. Em nosso caso, a melhor distribuição geométrica é formada pelos nós com ângulos mais distantes entre si e não colineares ao nó que deseja estimar sua posição via multilateração. A Figura 2 ilustra esse processo.

Por fim, é importante salientar que a seleção de nós acontece em duas etapas. A primeira etapa ocorre assim que um novo beacon é recebido. Se este beacon respeita as restrições dos ângulos e o número máximo de saltos (medido em Time to Live - TTL), este é atualizado e/ou acrescentado na lista de nós vizinhos. A segunda etapa ocorre no processo de Cooperative Positioning, que possui o objetivo de encontrar a melhor combinação de nós para multilateração com base nos valores de GDOP.

Esta estratégia de seleção de nós é um passo importante para a solução Cooperative Positioning. O Algoritmo 3 descreve a seleção de nós que ocorre ao receber um novo beacon. O Algortimo 4 descreve a solução Cooperative Positioning proposta onde $C C P o s$ é a posição final estimada via multilateração com a combinação de nós de GDOP mínimo. drModule representa o módulo da solução DR. Como último passo da solução proposta, a próxima seção apresenta a correção da posição do veículo considerando o 
mapa da via.

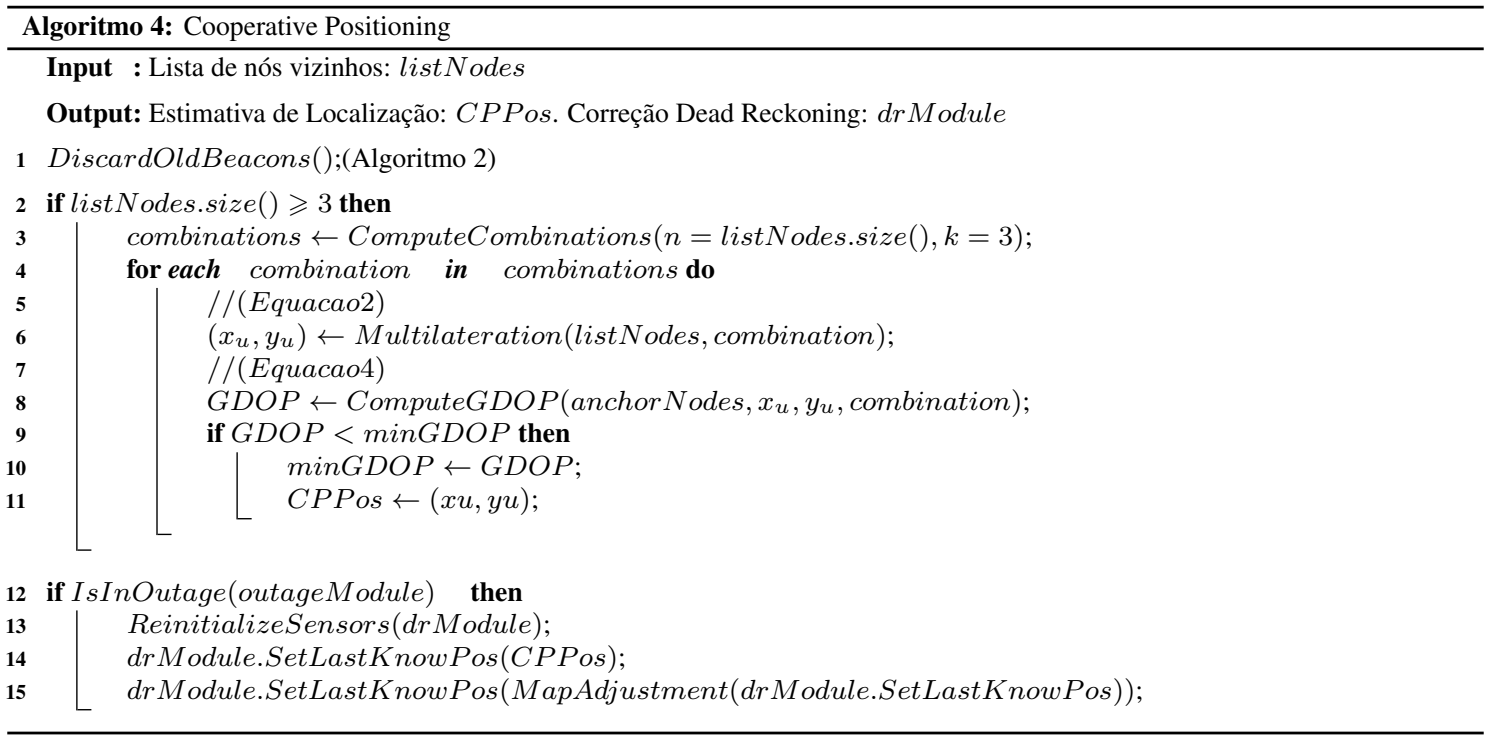

\subsection{Map Adjustment}

Com as informações de um mapa digital, foi definido um grafo $G(V, E)$ a partir do conjunto de pontos que formam as pistas dos túneis no mapa digital. As informações do mapa são fornecidas em coordenadas geográficas. Portanto, foi realizada uma conversão para o sistema de coordenas UTM [Zogg 2007] com o auxílio da biblioteca proj4² e realizamos os cálculos neste sistema de coordenas. A conversão para o sistema de coordenas UTM é necessária porque o simulador de tráfego utilizado pelas soluções de localização também utiliza o sistema de coordenadas UTM. Dada uma estimativa de posicionamento $(x, y)$, no sistema de coordenas UTM, a estimativa de posicionamento $P_{E S T}$ considerando o mapa digital da via é realizada como descrito a seguir.

Seja o par de vértices $(P, Q) \in E$ do grafo com coordenadas $P\left(x_{1}, y_{1}\right), Q\left(x_{2}, y_{2}\right)$ e uma estimativa de posicionamento $P_{E S T}\left(x_{3}, y_{3}\right)$. A equação da reta que passa por $(P, Q)$ é definida por $R=P+u(Q-P)$. Na Figura 3 o ponto $P_{E S T}$ é mais próximo à reta $\mathrm{R}$ exatamente em sua tangente. O produto interno da tangente e a reta é 0 e, portanto, $\left(P_{E S T}-R\right) \cdot(Q-P)=0$. Segue que, $u=\frac{\left(x_{3}-x_{1}\right)\left(x_{2}-x_{1}\right)+\left(y_{3}-y_{1}\right)\left(y_{2}-y_{1}\right)}{\|Q-P\|^{2}}$.

Substituindo $u$ na equação da reta obtemos o ponto de intersecção de coordenadas no ponto $I(x, y)$ da tangente que pode ser descrito como $x=x_{1}+u\left(x_{2}-x_{1}\right)$ e $y=$ $y_{1}+u\left(y_{2}-y_{1}\right)$.

Se $u$ possui valor no intervalo $(0,1]$, então existe solução. Caso contrário, não existe um ponto tangente ao ponto $P_{E S T}$ entre os pontos $P, Q$. Com o objetivo de realizar um ajuste a geometria das pistas, esse processo é aplicado em todos os pares consecutivos $(P, Q)$ do grafo até encontrar uma solução que satisfaça as condições mencionadas anteriormente.

\footnotetext{
${ }^{2} \mathrm{http} / /$ proj4.org/
} 


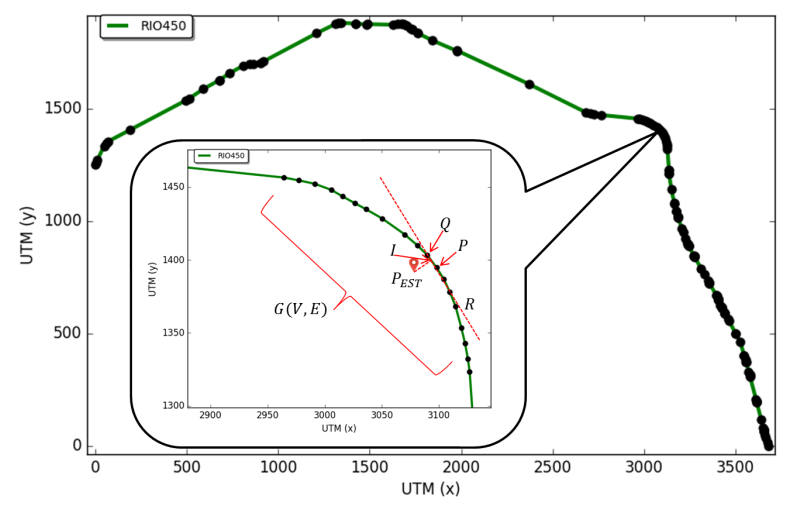

Figure 3. Ajuste as vias de tráfego por meio de uma mapa digital avançado.

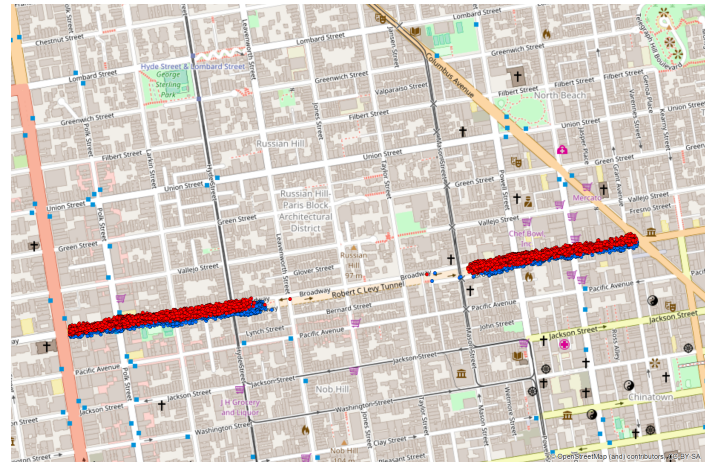

(a) Robert C Levy

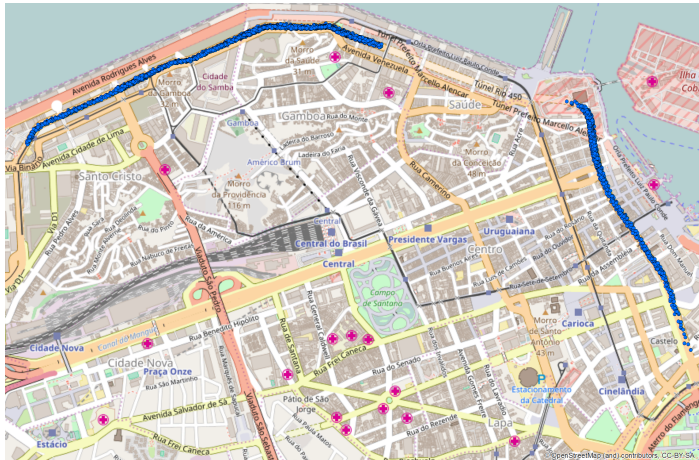

(b) Rio450

Figure 4. Caracterização dos túneis (a) Robert C. Levy, em São Francisco - EUA, e (b) Rio450 no Rio de Janeiro. Os pontos em vermelho e azul representam quedas de GPS em sentidos diferentes.

\section{Resultados}

\subsection{Caracterização do Problema de Indisponibilidade do Sistema GPS}

Para identificar os problemas apresentados pelo sistema GPS, utilizamos localizações reais de GPS obtidas de conjuntos de dados caracterizados em nossos trabalhos anteriores [do Nascimento et al. 2016b] [do Nascimento et al. 2016a]. Os dados foram obtidos de rotas de taxis e ônibus das cidades São Francisco (EUA), Dublin (Irlanda) e Rio de Janeiro. A partir dos conjuntos de dados, foram selecionadas regiões de interesse no entorno dos túneis Robert C. Levy e Rio450, das cidades de São Francisco e Rio de Janeiro, respectivamente. Ambos os túneis são urbanos e possuem comprimento diferentes. $\mathrm{O}$ primeiro possui 600 metros de extensão, enquanto o segundo é de 1480 metros. A escolha desses túneis tem por objetivo avaliar o impacto da solução proposta de posicionamento cooperativo em diferentes zonas de indisponibilidade do GPS.

A Figura 4 apresenta o resultado da caracterização para ambos túneis. Para cada ponto de queda em uma entrada do túnel ha um ponto correspondente na saída. As quedas acontecem não somente na imediata entrada e saída dos túneis, mas em toda a região entorno dos túneis. Isso ocorre devido ao fato de o receptor GPS possuir um atraso de sincronização no processo de perda e recuperação de sua linha de visada com os satélites. Maiores detalhes da caracterização podem ser encontrados em [do Nascimento et al. 2016b] e [do Nascimento et al. 2016a]. 


\subsection{Ambiente de Simulação}

Com objetivo de avaliar o desempenho da solução proposta, simulações foram realizadas com o framework Veins versão 4.5 [Sommer et al. 2011] que acopla o simulador de tráfego de veículos $\mathrm{SUMO}^{3}$ versão 0.29 e o simulador de rede OMNET++ 5.0 ${ }^{4}$. Com esta suíte de simuladores é possível definir todos os parâmetros da mobilidade dos veículos e todos os parâmetros da rede veicular de acordo com o padrão IEEE 802.11p. Para cada queda de GPS registrada no processo de caracterização [do Nascimento et al. 2016b] [do Nascimento et al. 2016a], sumarizado na Seção 5.1, foi adiconado um veículo no ambiente de simulação que reproduz a situação de indisponibilidade do GPS e utiliza a solução proposta.

O modelo de mobilidade Krauß Model foi utilizado nas simulações, no qual os veículos foram configurados com $50 \%$ de variação[Ros et al. 2014]. Isto significa que os veículos vão possuir diferentes comportamentos quanto a aceleração, desaceleração e escolha das vias de tráfego. Os parâmetros do giroscópio para a abordagem Dead Reckoning foram obtidos com base no datasheet de um giroscópio automotivo desenvolvido pela Bosch [Robert Bosch GmbH 2015]. Os parâmetros de simulação dos veículos e da rede estão sumarizados nas Tabelas 1 e 2.

\begin{tabular}{cc}
\hline Parâmetro & Valor \\
\hline \hline Modelo de Mobilidade & Krauß Model \\
Comprimento & $3.5(\mathrm{~m})$ \\
Aceleração & $1.0\left(\mathrm{~m} / \mathrm{s}^{2}\right)$ \\
Velocidade & {$[10.5,20.1](\mathrm{m} / \mathrm{s})$} \\
Variação & 0.5 \\
Erro GPS RIO 450 & $\mu_{R I O 450}=9.50$, \\
& $\sigma_{R I O 450}=3.42$ \\
Erro GPS RCLT & $\mu_{R C L T}=6.13$, \\
& $\sigma_{R C L T}=3.21$ \\
Frequência GPS & $10 H z$ \\
ARW Gyro & $0.063245^{\circ} / \mathrm{s} / \mathrm{sqrt}(\mathrm{Hz})$ \\
Sensitividade Gyro & $\pm 2 \%$ \\
Frequência Gyro & $10 \mathrm{~Hz}$ \\
\hline
\end{tabular}

Table 1. Parâmetros dos Veículos.

\begin{tabular}{cc}
\hline Parâmetro & Valor \\
\hline \hline Tecnologia Sem-Fio & $W A V E I E E E 802.11 p$ \\
Raio de Comunicação & $300 \mathrm{~m}$ \\
Taxa de Transmissão & $6 \mathrm{Mbit} / \mathrm{s}$ \\
Path Loss Model & log normal shadowing \\
Path Loss Parameters & $P L_{0}=53.57 \mathrm{~dB}, \alpha=1.77 \mathrm{~dB}$, \\
& $\sigma_{S}=3.36 \mathrm{~dB}$ \\
Frequência de beacons & $10 \mathrm{~Hz}$ \\
$\mathrm{~N}^{\circ}$ Max. Saltos (TTL) & 4 \\
Tráfego de veículos & 1200 Vei./h(1vei.acada3seg.) \\
\hline
\end{tabular}

Table 2. Parâmetros da Rede.

\subsection{Resultados}

Todos os resultados apresentados nesta Seção foram obtidos a partir de 33 simulações que seguem uma distribuição T Student com intervalo de confiança de 95\%. As Figuras 5 e 6 apresentam os resultados das reproduções das quedas de GPS e utilização da solução Cooperative Positioning proposta. Uma queda arbitrária de cada túnel foi selecionada para avaliar o impacto das características de cada cenário na solução proposta. São apresentados os resultados da trajetória e erro de posicionamento.

As linhas em vermelho, verde, magenta e ciano representam respectivamente as trajetórias e erros das solução GPS stand-alone (GPS), GPS integrado com Dead Reckoning e Cooperative Positiong (GPS-DR-CP), e a solução anterior com Map Adjustment (GPS-DR-CP-MA). É possível observar que o efeito random walk do giroscópio contribui significativamente para a discrepância na estimativa Dead Reckoning em situações de indisponibilidade do GPS. De modo geral, o tempo de uso do Dead Reckoning sem nenhuma fonte de reinicialização acarreta em um evolução rápida do erro de posicionamento.

\footnotetext{
${ }^{3}$ sumo.dlr.de

${ }^{4}$ https://www.omnetpp.org/
} 
Ainda nas Figuras 5 e 6, é possível perceber melhorias consideráveis nas estimativas de posicionamento da solução Dead Reckoning. Devido aos reinícios providos pela solução Cooperative Positioning, as estimativas de posicionamento estão bem mais próximas da trajetória real do veículo. Na Figura 6(b) do túnel Robert C. Levy, os erros são mantidos abaixo de 20 metros na maior parte do tempo. Apenas por volta dos $110 \mathrm{se}-$ gundos da trajetória, o erro atinge um pico de 40 metros, mas é corrigido logo em seguida pela abordagem Cooperative Positioning. Na Figura 5(b) os erros também são mantidos a baixo dos 20 metros. No entanto, é possível observar um leve aumento no erro de posicionamento ao longo da trajetória. Isso se deve ao fato do túnel ser maior o que acarreta em um maior acúmulo de erros da solução Dead Reckoning.

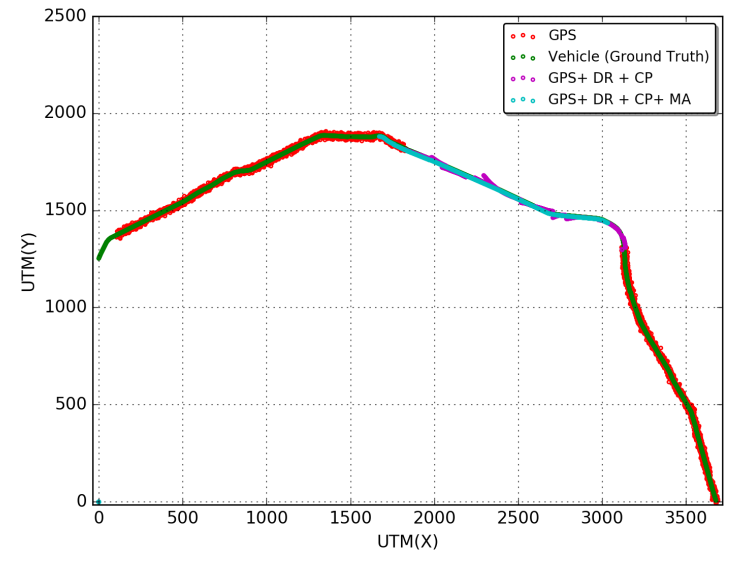

(a) Trajetória

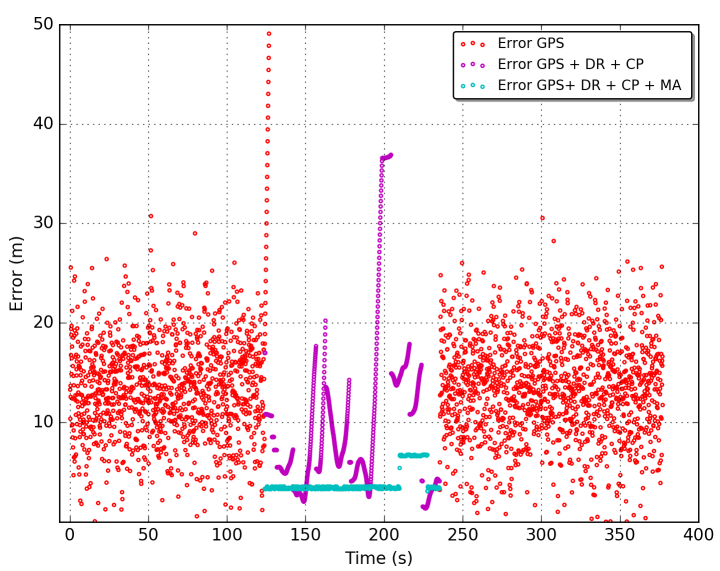

(b) Erro

Figure 5. Trajetória e Erro para o túnel Rio 450.

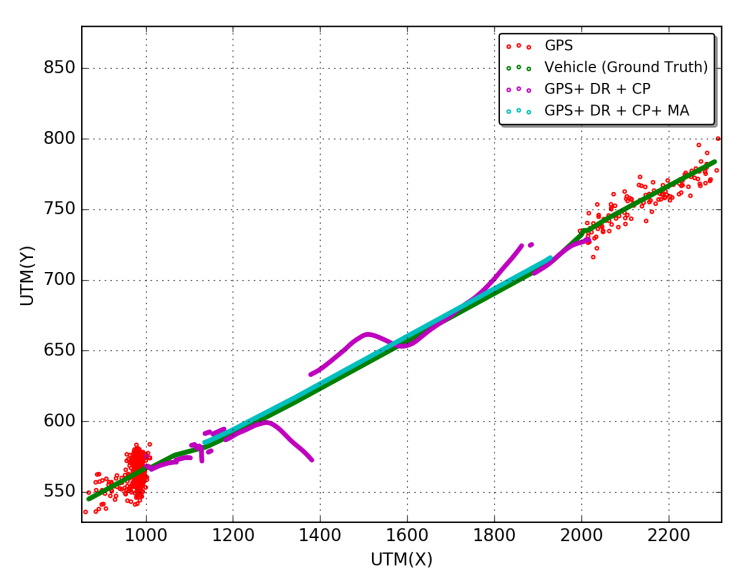

(a) Trajetória

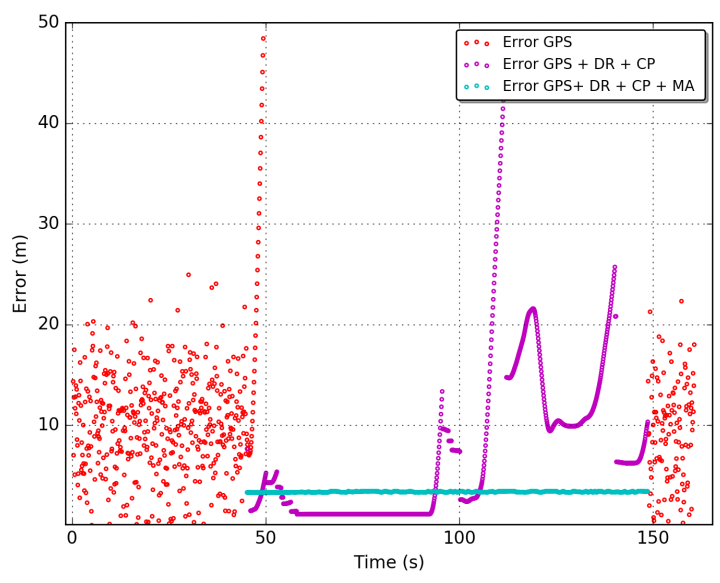

(b) Erro

Figure 6. Trajetória e Erro para o túnel Robert C. Levy.

Quando o Map Adjustment é utilizado para correção da estimativa final de posicionamento, os erros são ainda menores. São observados erros na faixa de 5 a 7 metros para o túnel Rio 450, e de aproximadamente 3 metros para o túnel Robert C. Levy. No entanto, a confiabilidade desse ajuste depende da confiabilidade que o mapa digital fornece quanto a geometria da pista na qual o veículo trafega. 
Com o objetivo de obter o comportamento geral da solução Cooperative Positioning Integrada ao Dead Reckoning, realizamos uma análise exploratória utilizando a métrica RMSE (Equação 5). Esta métrica é comumente utilizada na literatura para avaliar o erro das soluções de localização.

$$
R M S E=\frac{1}{n} * \sum_{i=0}^{n} \sqrt{\left(x_{i-v e h}-x_{i-e s t}\right)^{2}+\left(y_{i-v e h}-y_{i-e s t}\right)^{2}}
$$

As variáveis $\left(x_{i-v e h}, y_{i-v e h}\right)$ e $\left(x_{i-e s t}, y_{i-e s t}\right)$ representam a posição do veículo e a estimada utilizando GPS, GPS+DR, GPS+DR+CP e GPS+DR+CP+MA. Para mostrar o impacto do tempo no erro da solução, dividimos os tempos de queda de cada veículo em níveis de tempos de indisponibilidade do GPS. Tempos variando de $10 \%$ a $100 \%$ do tempo total de queda foram relacionados com o RMSE.

As Figuras 7(a) 7(b) apresentam os resultados numéricos do RMSE de acordo com o tempo de indisponibilidade do GPS. Considerando os primeiros $10 \%$ do tempo total de indisponibilidade do GPS, as soluções GPS, GPS + DR, GPS+ DR + CP, GPS + DR + CP + MA apresentam os RMSEs 57, 13, 10 e 5 metros para o túnel RIO 450; e 96, 12, 7, 3 para o túnel RCLT. Para os demais níveis, o RMSE das soluções GPS e GPS + DR crescem proporcionalmente ao tempo total de queda.

No entanto, a solução GPS+DR+CP cresce até cerca de $50 \%$ do total de tempo da queda e decresce logo em seguida. Isto se deve ao fato que apenas os veículos fora do túnel enviam beacons e, portanto, o erro na região central do túnel é maior do que nas entradas e saídas. São observados RMSEs máximos de 17 e 12 metros para os túneis RIO 450 e RCLT. A solução GPS+DR+CP+MA mantém o RMSE constante para todas as quedas de GPS analisadas e aproximadamente 5 metros para o túnel RIO 450 e 3 metros para o túnel RCLT.

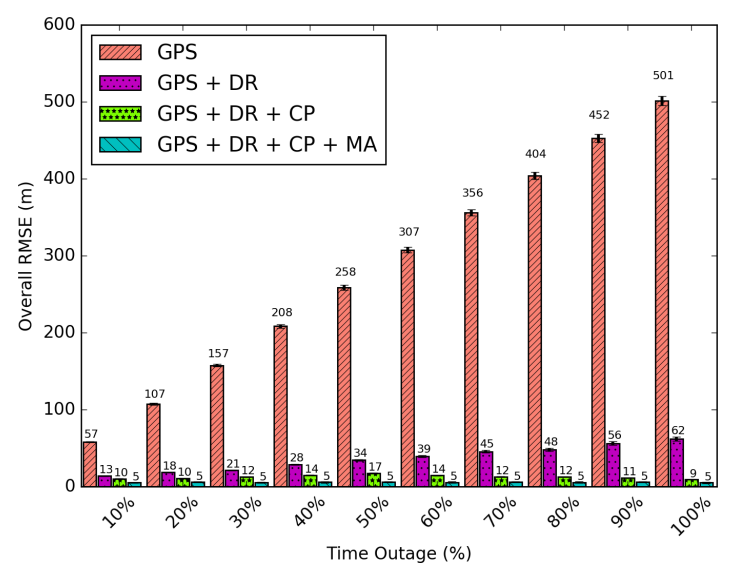

(a) Rio 450

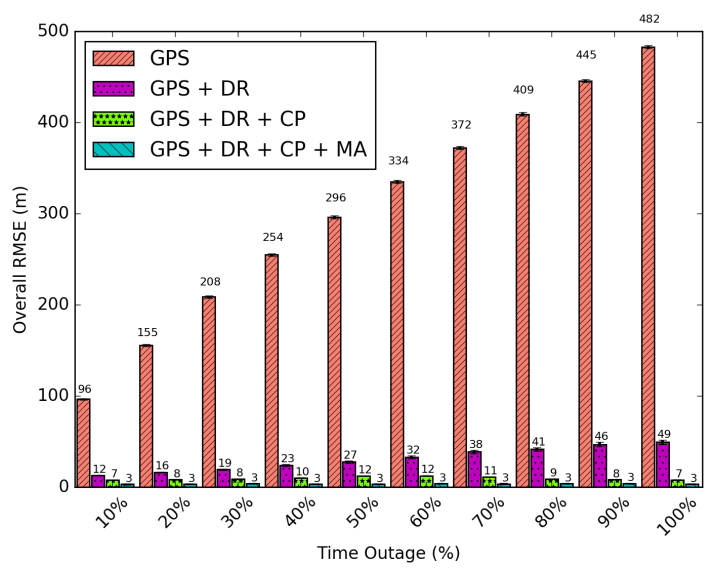

(b) RCLT

Figure 7. RMSE de acordo com porcentagens de tempos de indisponibilidade para cada técnica de localização e a solução final integrada.

Os ganhos das soluções Cooperative Positionig adiconada ao Dead Reckoning com e sem ajuste ao mapa, em relação as soluções GPS e GPS + DR podem ser obtidos pela Equação 6.

$$
\text { Ganho_Medio }=\frac{1}{10} * \sum_{t=10(\%)}^{100 \%}\left(\left(1-\frac{R M S E_{S_{1}}(t)}{R M S E_{S_{2}}(t)}\right) * 100\right)
$$


Onde, $S_{1}$ representa a solução GPS+DR+CP ou GPS+DR+CP+MA e $S_{2}$ as demais soluções comparadas (GPS, GPS+DR, GPS+DR+CP). Em suma, o ganho representa a diminuição efetiva do RMSE ao se aplicar a integração das técnicas desenvolvidas.

Além de cobrir $100 \%$ da área de indisponibilidade, a solução GPS+DR+CP apresenta ganhos entre $96 \%$ e $98 \%$ em relação a solução GPS stand-alone e $58 \%$ e $63 \%$ quando comparado com a solução GPS+DR. Quando o ajuste às vias de tráfego dos veículos é utilizado (GPS+DR+CP+MA), os ganhos são ainda mais efetivos: $97 \%$ a $98 \%$ em comparação ao GPS, $83 \%$ a $88 \%$ para GPS+DR e $59 \%$ a $67 \%$ de redução do RMSE em relação a solução GPS+DR+CP.

\section{Conclusões e Trabalhos Futuros}

Com objetivo de solucionar os problemas de indisponibilidade de GPS, neste trabalho foi desenvolvida e avaliada uma solução integrada de localização para as redes veiculares. Com base na capacidade de comunicação entre os veículos, a solução proposta integra a técnica de localização Dead Reckoning com uma solução Cooperative Positioning baseada na diluição da precisão de localização, que é drasticamente afetada pela geometria dos nós (GDOP). Além disso, com o suporte de um mapa digital, um ajuste de posicionamento é efetuado com base na geometria das pistas onde os veículos trafegam. A solução integrada de localização foi avaliada no cenário de túneis, onde zonas de GPS sem linha de visada foram caracterizadas a partir de datasets reais de GPS.

Resultados de simulação mostram a viabilidade da solução proposta. Além de cobrir $100 \%$ da área de indisponibilidade do GPS, a solução apresenta RMSEs na faixa de 3 a 5 metros. Em comparação com as soluções GPS stand-alone e GPS+DR, o RMSE é reduzido entre $96 \%$ a $98 \%$ e $58 \%$ a $63 \%$. Com o ajuste às vias de tráfego, o RMSE é reduzido entre $97 \%$ a $98 \%$ em comparação ao GPS, $83 \%$ a $88 \%$ para GPS+DR e $59 \%$ a $67 \%$ de redução em relação a solução GPS+DR+CP. Como trabalho futuro, pretende-se utilizar filtro de Kalman para predição e fusão dos dados de localização.

\section{Agradecimentos}

Os autores agradecem o apoio financeiro da Fundação de Amparo à Pesquisa do Estado de São Paulo (FAPESP) através dos processos 2015/07538-1, 2015/24494-8, 2015/18808-0, do Conselho Nacional de Desenvolvimento Científico e Tecnológico (CNPq) processos 401802/2016-7 e 132244/2016-0 e da Fundação de Amparo à Pesquisa do Estado de Minas Gerais (FAPEMIG) processo APQ-03120-17.

\section{References}

Alam, N. and Dempster, A. G. (2013). Cooperative Positioning for Vehicular Networks: Facts and Future. IEEE Transactions on Intelligent Transportation Systems, 14(4):1708-1717.

Alotaibi, M. M., Boukerche, A., and Mouftah, H. (2014). Distributed relative cooperative positioning in Vehicular Ad-Hoc Networks. In 2014 Global Information Infrastructure and Networking Symposium (GIIS), pages 1-8. IEEE.

Boukerche, A., Oliveira, H. A., Nakamura, E. F., and Loureiro, A. A. (2008). Vehicular Ad Hoc Networks: A New Challenge for Localization-Based Systems. Computer Communications, 31(12):2838-2849.

do Nascimento, P. P. L. L., Pazzi, R. W., Guidoni, D. L., and Villas, L. A. (2016a). A Geodesic Dead Reckoning Solution for Vehicular Networks. In 2016 8th IEEE LatinAmerican Conference on Communications (LATINCOM), pages 1-6. IEEE. 
do Nascimento, P. P. L. L., Pazzi, R. W., Guidoni, D. L., and Villas, L. A. (2016b). Characterizing GPS outages: Geodesic Dead Reckoning solution for VANETs and ITS. In 2016 IEEE 15th International Symposium on Network Computing and Applications (NCA), pages 18-21. IEEE.

Drawil, N. M. and Basir, O. (2010). Intervehicle-Communication-Assisted Localization. IEEE Transactions on Intelligent Transportation Systems, 11(3):678-691.

Hoang, G.-M., Denis, B., Harri, J., and Slock, D. T. M. (2017). Robust data fusion for cooperative vehicular localization in tunnels. In 2017 IEEE Intelligent Vehicles Symposium (IV), pages 1372-1377. IEEE.

Lawlor, J. (1991). Auto Math Handbook: Calculations, Formulas, Equations and Theory for Automotive Enthusiasts. Penguin Publishing Group.

OMS (2015). Global Status Report on Road Safety 2015. Technical report, World Health Organization.

Parker, R. and Valaee, S. (2007). Vehicular node localization using received-signalstrength indicator. IEEE Transactions on Vehicular Technology, 56(6 I):3371-3380.

Robert Bosch GmbH (2015). SMI130 Automotive Electronics.

Rohani, M., Gingras, D., Vigneron, V., and Gruyer, D. (2015). A New Decentralized Bayesian Approach for Cooperative Vehicle Localization Based on Fusion of GPS and VANET Based Inter-Vehicle Distance Measurement. IEEE Intelligent Transportation Systems Magazine, 7(2):85-95.

Ros, F. J., Martinez, J. A., and Ruiz, P. M. (2014). A survey on modeling and simulation of vehicular networks: Communications, mobility, and tools. Computer Communications, 43:1-15.

Sauer, T. (2011). Numerical Analysis. Addison-Wesley Publishing Company, 2nd edition.

Skog, I. and Handel, P. (2009). In-Car Positioning and Navigation Technologies - A Survey. IEEE Transactions on Intelligent Transportation Systems, 10(1):4-21.

Sommer, C., German, R., and Dressler, F. (2011). Bidirectionally Coupled Network and Road Traffic Simulation for Improved IVC Analysis. IEEE Transactions on Mobile Computing, 10(1):3-15.

Sun, L., Wu, Y., Xu, J., and Xu, Y. (2012). An RSU-assisted localization method in non-GPS highway traffic with dead reckoning and V2R communications. 2012 2nd International Conference on Consumer Electronics, Communications and Networks, CECNet 2012 - Proceedings, pages 149-152.

Swaszek, P. F., Hartnett, R. J., Seals, K. C., and Swaszek, R. M. A. (2017). A Temporal Algorithm for Satellite Subset Selection in Multi-Constellation GNSS. In Precise Time and Time Interval Meeting, Monterey, CA.

Wahab, A. A., Khattab, A., and Fahmy, Y. a. (2013). Two-way TOA with limited dead reckoning for GPS-free vehicle localization using single RSU. 2013 13th International Conference on ITS Telecommunications, ITST 2013, pages 244-249.

Woodman, O. J. (2007). An introduction to inertial navigation. Technical report, University of Cambridge, Cambridge.

Zogg, J. M. (2007). GPS - Essentials of Satellite Navigation. UBLOX. 